Background: Subjective sleep problems, including difficulties falling asleep, waking up, un-restorative sleep and daytime sleepiness are highly prevalent in patients with juvenile fibromyalgia (JFM). Sleep disturbances has been considered a consequence of severe pain and depression, but also in healthy individuals sleep deprivation is also a risk factor for the development of chronic widespread pain, tenderness and fatigue, suggesting the important role of sleep in pain control and in the pathophysiology of fibromyalgia.

Objectives: To estimate the incidence of polysomnographic alterations in JFM and to explore the relationship between sleep problems and the musculoskeletal pain, fatigue and mood and anxiety disorders.

Methods: 21 patients (M 3; F 18; mean age 16,1) with JFM were included. The objective sleep quality was measured by overnight polysomnography (PSG) (using the EMBLETTA MPR PG device). PSG data were compared to age and sex-matched controls. The subjective sleep disturbances were assessed by the Sleep Condition Indicator (SCI). Musculoskeletal symptoms were evaluated by using the widespread pain index (WPI). Pain intensity was evaluated on a 0-10 visual analogical scale (PVAS). Fatigue was assessed by using the Symptom Severity (SS) questionnaire. Mood and anxiety disorders were evaluated by using the Children Depression Index (CDI) and the Multidimensional Anxiety Scale for Children (MASC). Comparison of categorical data was performed by means of the Fisher's Exact test. The relationship between sleep quality and clinical symptoms were assessed using Spearman's rank order correlation coefficient ( $r s$ ). All statistical test were 2 -sided and $p$ values less than 0.05 were considered statistically significant.

Results: Nineteen out of $21(90.5 \%)$ patients complained subjective sleep disturbances and un-restorative sleep. Seven out of 21 (33.3\%) patients had mood and anxiety disorders. Eight out of 21 patients (38.1\%) showed an electroencephalographic pattern of alpha wave intrusion in slow wave sleep (SWS). SCI was significantly correlated to CDI score rs $-0,775(p \leq 0,0001)$, MASC $0,61(p=0,005)$, WPI -0,731 ( $p=0,001)$, SSI 0,492 $(p=0,038)$, PVAS -0,590 $(p=0,006)$.

Conclusion: A substantial percentage of JFM patients experience sleep disturbances, which are, correlated with the severity of the muscolskeletal sympotms and mood and anxiety disorders. One third of JFM patients have alpha intrusion in the SWS. The important role of sleep in pain control suggests that the development of treatments to improve sleep quality may lead to more effective management of fibromyalgia in the future.

References:

[1] Ting TV et. al 2010 American College of Rheumatology Adult Fibromyalgia Criteria for Use in an Adolescent Female Population with Juvenile Fibromyalgia. J Pediatr. 2016 Feb;169:181-7.

[2] Choy $\mathrm{EH}$. The role of sleep in pain and fibromyalgia. Nat Rev Rheumatol. 2015;11:513-20.

[3] Roizenblatt $S$ et al. Alpha sleep characteristics in fibromyalgia. Arthritis Rheum. 2001;44, 222-230.

Disclosure of Interests: None declared

DOI: 10.1136/annrheumdis-2020-eular.6112

\section{AB0993 A CASE SERIES OF KAWASAKI DISEASE FROM KENYA}

\section{Njeru ${ }^{1}$, A. Migowa ${ }^{1} .{ }^{1}$ Aga Khan Hospital, Nairobi, Kenya}

Background: Kawasaki disease (KD) has been described across the globe, including the African continent, but none yet from Kenya.

Objectives: To describe the clinical features and management strategies of pediatric patients diagnosed with Kawasaki Disease at a tertiary referral hospital in Kenya.

Methods: A retrospective chart review was undertaken for the period January 2013 to December 2017 for all pediatric patients admitted at Aga Khan University Hospital Nairobi, Kenya. All medical records with a discharge diagnosis of Kawasaki disease were reviewed, de-identified and data extracted using a data collection tool.

Results: Among the 15 cases identified, 8 (53\%) had complete KD. The mean age was 1 year 10 months with a slight increase in males (53.3\%). The mean duration of symptoms at diagnosis was 7.2 days (range 1-11 days). Fourteen patients $(93.3 \%)$ received both intravenous immunoglobulin and aspirin but dosing varied from high dose aspirin $(80-90 \mathrm{mg} / \mathrm{kg} /$ day) to low dose aspirin $(3 \mathrm{mg} / \mathrm{kg} /$ day). Baseline cardiac evaluations were done among these 14 $(93.3 \%)$ and one patient was found to have bilateral dilated coronaries. Only 5 patients (33.3\%) had repeat echo examinations within 6 weeks after diagnosis all of which were normal.

Conclusion: The challenges faced in the management of KD in Kenya include awareness of the disease, access and expertise to pediatric echocardiography, follow-up, access and cost of IVIG. Increasing awareness and improving health care resources is important in improving outcomes of KD in Kenya.

Keywords: Kawasaki Disease, Pediatric Rheumatology, Kenya, Global Health, Vasculitis

References:
[1] McCrindle BW, Rowley AH, Newburger JW, Burns JC, Bolger AF, Gewitz M, Baker AL, Jackson MA, Takahashi M, Shah PB, Kobayashi T, Wu MH, Saji TT, Pahl E. Diagnosis, Treatment, and Long-Term Management of Kawasaki Disease: A Scientific Statement for Health Professionals From the American Heart Association. Circulation. 2017;135(17):e927-e999.

[2] Kawasaki T. Acute febrile mucocutaneous syndrome with lymphoid involvement with specific desquamation of the fingers and toes in children. Arerugi (Japanese) 1967;16:178-222.

[3] Singh S, Sharma A, Jiao F. Kawasaki Disease: Issues in Diagnosis and Treatment - A Developing Country Perspective. Indian J Pediatr 2016:83(2):140-145.

[4] Scuccimarri R. Kawasaki Disease. Pediatr Clin N Am 2012; 59:425-445.

Disclosure of Interests: None declared

DOI: 10.1136/annrheumdis-2020-eular.6345

\section{\begin{tabular}{|l|l}
\hline AB0994 NEW ALTERNATIVE IN THE TREATMENT OF PATIENT \\
\hline
\end{tabular} WITH MUTATION OF GEN LACC1}

R. M. Alcobendas ${ }^{1}$, C. Quintana ${ }^{1}$, J. Arostegui ${ }^{2}$, C. Udaondo ${ }^{1}$, S. Murias Loza ${ }^{1}$, A. Remesal'. ' University Hospital La Paz, Madrid, Spain; ${ }^{2}$ Clinic Hospital, Barcelona, Spain

Background: Few patients have been described in the literature with mutations in the Lacasa Domain containing one (LACC1) gene. Its clinical presentation usually associates sustained systemic inflammation associated with chronic polyarticular erosive arthritis. Until now, there have been multiple treatments described to try to control the disease, however, they are generally unsuccessful in the long term.

Objectives: Describe the clinical course of a patient as well as the different treatments used

Methods: Clinical chart review

Results: Female 18-year-old born from a consanguineous Moroccan couple. Mother, brother and sister with similar conditions. She started at 3 years with fever, anemia, intense elevation of acute phase reactants and symmetric polyarthritis (knees, elbows, carps, shoulders, hands and ankles). Subsequen whole exome sequencing identified c.128_129deIGT mutation in the LACC1/ FAMIN gene. During the course of her illness, she has received treatment with oral, intravenous and infiltrated corticosteroid, methotrexate and etanercept, without getting adequate control of the disease. In 2016, she started treatmen with tocilizumab ( $8 \mathrm{mg} / \mathrm{kg}$ every two weeks), obtaining an acceptable control of the disease (requiring periodic infiltrations every 2-3 months due to persistent arthritis). Nonetheless, in April 2019, she consulted for clinical worsening of the arthritis and laboratory test (C reactive protein $99.7 \mathrm{mg} / \mathrm{L}$, erythrosedimentation rate $53 \mathrm{~mm} / \mathrm{h}$, leukocytes $13,500 / \mu \mathrm{L}$ and neutrophils $10,930 / \mu \mathrm{L}$ ). At that time, she discontinued therapy with tocilizumab and started tofacitinib $5 \mathrm{mg}$ every 12 hours with good evolution. Since its introduction, it has not required joint infiltration again and the inflammatory parameters (persistently elevated previously) have normalized.

Conclusion: The jak kinasa inhibitors may be a treatment option in those patients with bad response to conventional therapy.

\section{References:}

[1] Rabionet R, Remesal A, Mensa-Vilaró A, Murías S, Alcobendas R, González-Roca E, Ruiz-Ortiz E, Antón J, Iglesias E, Modesto C, Comas D, Puig A, Drechsel O, Ossowski S, Yagüe J, Merino R, Estivill X, Arostegui J. Biallelic loss-of-function LACC1/FAMIN Mutations Presenting as Rheumatoid Factor-Negative Polyarticular Juvenile Idiopathic Arthritis. Sci Rep. 2019 Mar 14;9(1):4579

Disclosure of Interests: None declared

DOI: 10.1136/annrheumdis-2020-eular.5555

\section{AB0995 \\ JUVENILE ONSET SYSTEMIC LUPUS ERYTHEMATOSUS WITH SJÖGREN'S SYNDROME: CLINICAL AND LABORATORY FEATURES.}

M. Kaleda ${ }^{1}$, I. Nikishina ${ }^{2} .{ }^{1}$ V. A. Nasonova Research Institute of Rheumatology, Pediatric Department, Moscow, Russian Federation; ${ }^{1}$ V. A. Nasonova Research Institute of Rheumatology, Pediatric Department, Moscow, Russian Federation

Background: Systemic lupus erythematosus with juvenile onset (jSLE) with Sjogren's syndrome (SS) in children is a poorly studied and rare combination, the frequency of which, according to the literature, is $7.5-10.0 \%{ }^{1}$.

Objectives: To study demographic data, specific features of jSLE with SS in single center.

Methods: Retrospective study of all consequently patients (pts) of single-center in pediatric department with combination of jSLE and SS.

Results: SS was verified in 14 pts with jSLE (14.3\% were boys), which amounted to $15.5 \%$ of all pts with jSLE. The median age of jSLE onset was 13.5 y.o. [9.3; 14.9] The median of disease duration at the time of SS verification was $1.3 \mathrm{y}[0.6 ; 2.9]$. 